ISSN 2590-9770

The Art of Discrete and Applied Mathematics 2 (2019) \#P2.09

https://doi.org/10.26493/2590-9770.1332.594

(Also available at http://adam-journal.eu)

\title{
Open problems from NCS 2018
}

\author{
Jonathan Leech \\ Westmont College Santa Barbara, CA, USA \\ João Pita Costa * \\ Jožef Stefan Institute, Ljubljana, Slovenia
}

Received 14 July 2019, accepted 19 September 2019, published online 31 December 2019

\begin{abstract}
All participants in the workshop NCS 2018 were invited to submit a list of open problems, typically problems relevant to the subject matter of their talk. These problems follow, grouped according the particular individual presenting them. These individuals appear with their problem sets in alphabetical order, the sole exception being that the Honoree of the workshop appears first. Some of the presenters give a bit of background to accompany their problems. In other cases, such as myself, the presenter assumes that enough background was given in their talk as published herein. Thus the reader is invited to refer back to the relevant article. It is hoped that in the months to follow some of these problems will be solved, or at least, considerable light will be shed on them.

Keywords: Skew lattice, ring, primitive skew lattice, dual congruence distributivity, orthodox semigroup, sheaf representation, left regular band, quasilattice, paralattice, covering lattice, skew Boolean algebra, congruence lattice, antilattice, skew Heyting algebra, coset structure, comodernistic lattice.
\end{abstract}

Math. Subj. Class.: 06A75, 06B20, 06B75

\section{Jonathan Leech}

Problem 1.1. The following problem considers skew lattices in the context of rings.

(1) Given a finite left (right) regular band $B$, must the skew lattice generated from $B$ in the semigroup ring $\mathbb{Z}(B)$ be finite also?

(2) If $B$ is the free left (right) regular band on generators, $x_{1}, x_{2}, \ldots, x_{n}$, what must its generated skew lattice in $\mathbb{Z}(B)$ look like... in detail?

*The author thanks the support of the Croatian Science Foundation's funding of the project EVOSOFT, with the reference UIP-2014-09-7945.

E-mail addresses: leech@westmont.edu (Jonathan Leech), joao.pitacosta@ijs.si (João Pita Costa) 
The $\mathbb{Z}$-coefficient case has implications for the case of an arbitrary commutative ring $K$ with identity being used as the coefficient ring. Of course, if the generated skew lattice in (2) is finite for all $n$, then the answer to (1) is YES. In this case, for any finite left regular band in any ring, the generated skew lattice is finite.

Problem 1.2. Find necessary and sufficient conditions for a skew lattice $S$ to be embedded into a ring. That is for $S$ to have an isomorphic copy consisting of idempotents in a ring with the operations being multiplication and either the quadratic or cubic join. Preconditions include: being distributive (and thus categorical); being cancellative (and thus symmetric). Clearly skew Boolean algebras and their duals can be embedded into a ring. More generally, strongly distributive skew lattices [satisfying $x \wedge(y \vee z)=(x \wedge y) \vee(x \wedge z)$ and $(x \vee y) \wedge z=(x \wedge z) \vee(y \wedge z)]$ and dual strongly distributive lattices can be embedded into a ring. Are there other sufficient conditions?

Problem 1.3. In particular, find necessary and sufficient conditions for a primitive skew lattice to be embedded into a ring. A primitive skew lattice is both distributive and cancellative, Perhaps all primitive skew lattices can be so embedded. If not, then a third general requirement for a skew lattice to be embedded into a ring is that all of its primitive subalgebras can be so embedded. More generally, find necessary and sufficient conditions for skew chains $A>B>C$ or $A>B>C>D$ or longer, to be so embedded.

Problem 1.4. What can be said about the subvariety of skew lattices generated from all primitive skew lattices? It must be: distributive (and thus categorical), cancellative (and thus symmetric) and strictly categorical. What else? Again, what are necessary and sufficient conditions for a skew lattice to be in this subvariety?

Problem 1.5. What can one say about the subvariety of skew lattices generated jointly from the varieties of normal and conormal skew lattices?

\section{Robert J. Bignall}

Problem 2.1. Find a Mal'cev condition (see [21]) for dual congruence distributivity at a constant.

Problem 2.2. Characterise the class of dual binary discriminator varieties that are dually congruence distributive at their discriminating constant.

\section{Background}

Let $\mathrm{K}$ be a class of algebras of some given similarity type and let $\mathbf{0}$ be a constant term. Given an algebra $\mathbf{A} \in \mathrm{K}$ and $\Theta \in$ Con $\mathbf{A}$, denote the congruence class $\{a \in A \mid a \Theta 0\}$ by $[0] \Theta$, where $0=\mathbf{0}^{\mathbf{A}}$. $\mathbf{A}$ is said to be congruence distributive at $\mathbf{0}$, or congruence 0-distributive if for all $\Theta, \Psi, \Phi \in$ Con $\mathbf{A}$

$$
[0](\Theta \vee \Psi) \wedge \Phi=[0](\Theta \wedge \Phi) \vee(\Psi \wedge \Phi)
$$

$\mathbf{A}$ is said to be dually congruence distributive at $\mathbf{0}$, or dually congruence $\mathbf{0}$-distributive if for all $\Theta, \Psi, \Phi \in$ Con $\mathbf{A}$

$$
[0](\Theta \wedge \Psi) \vee \Phi=[0](\Theta \vee \Phi) \wedge(\Psi \vee \Phi)
$$


The class $\mathrm{K}$ is congruence $\mathbf{0}$-distributive (resp. dually congruence $\mathbf{0}$-distributive) if every $\mathbf{A} \in \mathrm{K}$ is congruence $\mathbf{0}$-distributive (resp. dually congruence $\mathbf{0}$-distributive); see [2]. These properties are described as being ideal-theoretic, because $[0] \Theta$ is an ideal of $\mathbf{A}$ with respect to the constant $\mathbf{0}$.

Dual congruence $\mathbf{0}$-distributivity is a stronger condition than congruence $\mathbf{0}$-distributivity. For example, meet semilattices with $\mathbf{0}$, or more generally normal bands with $\mathbf{0}$, are congruence $\mathbf{0}$-distributive but not dually congruence $\mathbf{0}$-distributive. On the other hand, skew Boolean algebras are dually congruence $\mathbf{0}$-distributive, and hence are also congruence 0-distributive. Skew Boolean algebras, or more generally algebras in skew Boolean discriminator varieties, enjoy a number of other strong ideal-theoretic properties, despite the fact that their congruence lattices in general satisfy no lattice identities.

I. Chajda [2] has given a Mal'cev condition for the class of congruence $\mathbf{0}$-distributive varieties, modelled on Bjarni Jónsson's well-know Mal'cev condition for the class of congruence distributive varieties [11]. However, it would appear that no-one has as yet solved the harder problem of finding a Mal'cev condition for the class of dually congruence $\mathbf{0}$ distributive varieties (or dually congruence 0-distributive SP classes; that is classes of algebras that are closed under the taking of products, subalgebras and isomorphisms.) The congruence lattices of algebras in dual binary discriminator varieties do not in general have any special properties. However, the ideals of such algebras play a central role. Another significant ideal theoretic property is congruence permutability at $\mathbf{0}$. A class $\mathrm{K}$ of algebras with a constant $\mathbf{0}$ is congruence permutable at $\mathbf{0}$ if for any $\mathbf{A} \in \mathrm{K}$ and any $\Theta, \Phi \in$ Con $\mathbf{A}$,

$$
[0] \Theta \circ \Phi=[0] \Phi \circ \Theta .
$$

$\mathrm{K}$ is arithmetic at $\mathbf{0}$ if it is both congruence distributive and congruence permutable at $\mathbf{0}$, in which case it is also dually congruence distributive at $\mathbf{0 .}$.

The class of binary discriminator varieties has an entirely ideal-theoretic characterisation as that class of pointed varieties that are arithmetic at their constant term, have equationally definable principal ideals, and are generated by a sub-class of ideal simple members. A worthwhile but as yet incomplete research program would be to characterise each pointed discriminator variety sub-class of the class of dual binary discriminator varieties in terms of the ideal theoretic properties of its members.

\section{Des FitzGerald}

Problem 3.1. Let $A$ be an algebra and $S$ its monoid of endomorphisms. It is known that if $S$ has commuting idempotents then $A$ has the properties:

(RI) the intersection of two retracts of $A$ is also a retract,

(UR) to each retract of $A$ corresponds a unique idempotent with that range,

and their duals. Is the converse true? This was posed as Problem 7.4 of [10] where a proof of the forwards implication may be found.

Problem 3.2. My paper [5] necessarily included (in Section 2) a development of groupoids over a band of objects. It seemed likely that this topic had been considered already, but I have since found nothing directly comparable in the literature. Hence the problem: describe the class of orthodox semigroups which are obtained as groupoids over a band of objects in the manner shown there. 


\section{Background for 3.1}

First note that every homomorphism (written as a right mapping) in a variety has a factorisation $f=h j$ with $h$ surjective and $j$ injective. If there are $h: A \rightarrow R, j: R \rightarrow A$ such that $j h=1_{R}$, then $h$ is called a retraction (and in fact is surjective) and $j$ is a section (and is injective). $R$ is a retract of $A$ and the congruence $\rho_{h}:=h \circ h^{-1}$ is a coretract of $A$. Of course $R \cong A / \rho_{h}$, so the distinction between retracts and coretracts is solely whether we consider $R$ as a subalgebra or a quotient algebra. Put $f=h j$; then $j h=1_{R}$ is equivalent to $f^{2}=f$ (this is easy folklore).

Now (RI) and (UR) are as stated, but to unpick my little concealment, we could rephrase (UR) as: to each section $j: R \rightarrow A$ corresponds a unique right inverse $h: A \rightarrow R$. Then the duals are

(RI*) the join of two coretracts of $A$ is a coretract of $A$;

(UR*) each retraction $h: A \rightarrow R$ has a unique section (left inverse).

I know no reference for my claim that $\mathrm{CI}: S=\operatorname{End} A$ has commuting idempotents implies all of these; here's my proof.

Proof. Let two retracts of $A$ be determined (as $A f$ and $A g$ ) by $f, g \in S$ with $f=f^{2}$, $g=g^{2}$, and $f g=g f$. Then $(f g)^{2}=f g$ and $A f g$ is a retract (and $\rho_{f g}$ is a coretract). Clearly

$$
A f g=A g f \subseteq A f, A g
$$

and so $A f g \subseteq A f \cap A g$. But if $x \in A f \cap A g$ then $x=x f=x g=x f g$, so $x \in A f g$. This proves $A f \cap A g=A f g$, a retract, and establishes (RI). Similarly, any two coretracts of $A$ are of the forms $\rho_{f}$ and $\rho_{g}$, with $f, g$ idempotents of End $A$. Then $\rho_{f} \vee \rho_{g} \subseteq \rho_{f g}$ $\left(x f=x^{\prime} f \Rightarrow x f g=x^{\prime} f g\right.$, etc.) But if $x f g=x^{\prime} f g$, we have

$$
x \rho_{f} x f \rho_{g} x^{\prime} f \rho_{f} x^{\prime} \quad \text { and } \quad x \rho_{g} x g \rho_{f} x^{\prime} g \rho_{g} x^{\prime},
$$

and thus $\left(x, x^{\prime}\right) \in \rho_{f} \vee \rho_{g}$ whence $\rho_{f g} \subseteq \rho_{f} \vee \rho_{g}$. Thus $\rho_{f} \vee \rho_{g}=\rho_{f g}$, a coretract; so (RI*) holds.

Now for (UR). Suppose there are $f=f^{2}, g=g^{2}$ such that the retract $R=A f=A g$. Then for any $x \in A$, there are $y, z \in A$ such that $x f=y g, x g=z f$. Then

$$
\begin{aligned}
x f g & =y g=x f \\
& =x g f=z f=x g,
\end{aligned}
$$

and so $f=g$ and we see (UR). To (UR*): Suppose there is $h: A \rightarrow R$ and sections $j, k: R \rightarrow A$ such that $j h=k h=1_{R}$. Then $h j, h k$ are idempotents in End $A$ and we have in turn $h j h k=h k h j, h k=h j$, and $k=j$.

\section{Sam van Gool}

Problem 4.1. What is the relationship, if any, between the duality for sheaf representations of distributive-lattice-ordered algebras of Gehrke and van Gool [6] and the duality for strongly distributive skew lattices of Bauer, Cvetko Vah, Gehrke, van Gool, and Kudryavtseva [1]? Sheaves play prominent but apparently distinct roles in these two dualities; any connection between the two could be an interesting direction for further research. 


\section{Michael Kinyon}

\subsection{Left regular bands and left-handed skew lattices as ordered sets}

When I've given talks about skew lattices to nonexperts, one of the things that typically bothers members of the audience is that unlike lattices, skew lattices do not have a nice order theoretic definition.

There was one attempt to give such a characterization by Gerhardts [7], who studied a variety of noncommutative lattices he called Fastverbanden. They have an unintuitive definition, but it is not difficult to show that they are precisely what we now call lefthanded binormal (normal and conormal) skew lattices. Gerhardts attempted to characterize Fastverbanden entirely in terms of their natural partial order and their natural preorder. Unfortunately, [7] has at least one mistake in it. However, the definitions can be modified to get his idea to work. For simplicity, I will just focus on bands here instead of skew lattices.

The basic structure $(X, \preceq, \leq)$ consists of a set $X$ with a preorder $\preceq$ and a partial order $\leq$ which refines $\preceq$ (that is, $\leq \subseteq \preceq$ ). Let $\mathcal{L}$ denote the equivalence relation associated to $\preceq$. We assume the following:

$$
\text { If } x \mathcal{L} y \text { and } x, y \leq z \text {, then } x=y .
$$

For $a, b \in X$, an element $c \in X$ is said to be a lower bound of the pair $(a, b)$ if $c \leq a$ and $c \preceq b$. We say that $c$ is an infimum of $(a, b)$ if

(i) $c$ is a lower bound of $(a, b)$, and

(ii) if $x \preceq a, b$, then $x \preceq c$.

It is easy to prove that a pair $(a, b)$ can have at most one infimum. (The definition of infimum is where I differ from Gerhardts.)

A left normal band $(X, \leq, \preceq)$ (in the order theoretic sense) is a structure as above such that every ordered pair $(a, b)$ of elements has an infimum, which we denote by $a \wedge b$. We then can prove the expected theorem: left normal bands in the order theoretic sense are precisely the same as left normal bands in the algebraic sense.

It is easy to get the corresponding result for left-handed binormal skew lattices, and it is also not hard to generalize from the one-sided case to normal bands by using both the $\mathcal{L}$ and $\mathcal{R}$-preorders.

Left normality essentially comes from property $(*)$ above.

Problem 5.1. Generalize the above characterization to left regular bands. What property should replace $(*)$ ?

\subsection{Quasilattices and paralattices}

Recall that a double band $(X, \vee, \wedge)$ is a

- quasilattice if the natural preorders dualize each other,

- paralattice if the natural partial orders dualize each other.

Both classes of noncommutative lattices form varieties. Quasilattices are axiomatized by

$$
x \wedge(y \vee x \vee y) \wedge x=x=x \vee(y \wedge x \wedge y) \vee x,
$$


while paralattices are axiomatized by

$$
(x \vee y \vee x) \wedge x \wedge(x \vee y \vee x)=x=(x \wedge y \wedge x) \vee x \vee(x \wedge y \wedge x)
$$

(These are not the four identities axiomatizing paralattices in [12] but it is easy to see these two are equivalent to those.)

Let us say that a double band $(X, \vee, \wedge)$ is a _lattice if it satisfies the following implications:

$$
\begin{aligned}
& x \leq_{\wedge} y \quad \Rightarrow \quad y \preceq \vee x \\
& x \leq_{\vee} y \quad \Rightarrow \quad y \preceq \wedge x
\end{aligned}
$$

(Part of the problem here is to find a suitable name for this class of noncommutative lattices.) It is clear that -lattices include both quasilattices and paralattices as special cases. It is also straightforward to show that -lattices form a variety axiomatized by

$$
x \wedge(x \vee y \vee x) \wedge x=x=x \vee(x \wedge y \wedge x) \vee x .
$$

Problem 5.2 (Suggested by J. Leech). Describe the join variety of the varieties of paralattices and quasilattices.

\section{Jurij Kovič}

Problem 6.1. Define the concept of (and develop a theory for) a covering of a (skew) lattice. Use the analogy with covering graphs (voltages).

\section{Background}

The concept of a covering graph. Definitions of graphs, covering graphs and voltages are in detail explained in [15, pp. 96-97]. Covering graphs were first introduced in [9] for directed graphs, but they may be applied also to undirected graphs, as in [16]. Lifting graph automorphisms from the underlying graph is explained in [14].

\section{Ganna Kudryavtseva}

Some problems on skew Boolean algebras are proposed below. For a concise background on skew Boolean algebras, please refer to [13].

Problem 7.1. Study objects obtained by relaxations of the definition of an SBA $S$. What if normality is dropped and one requires only $S$ is symmetric, has 0 and $S / \mathcal{D}$ is a Boolean lattice? What if symmetricity is dropped and one requires only $S$ is normal, has 0 and $S / \mathcal{D}$ is a Boolean lattice? These, in each case, are generalizations of SBAs. Study their structure. Do they form a variety (in each case)? How are they related to SBAs?

Problem 7.2. Let $S$ be a LSBIA (left handed skew Boolean intersection algebra). Then $S^{*}$ is a (locally compact) Boolean space so that there is its dual GBA, $B$. Is it possible to extend the assignment $S \mapsto B$ to a functor? What are adjoints, if exist? What can be said about the relationship between $S / \mathcal{D}$ and $B$ ?

Problem 7.3. There is a forgetful functor from Boolean inverse semigroups to LSBAs (indeed, domain and range maps of an étale groupoids are étale maps). Does it have a left adjoint? Then how is it constructed? A similar question can be asked also about Boolean inverse $\wedge$-semigroups and LSBIAs. 


\section{Tomaž Pisanski}

Problem 8.1. Given a finite lattice $K$. Find a test that will tell whether $K$ is isomorphic to a congruence lattice $\operatorname{Con}(N)$ of some antilattice.

Problem 8.2. Any automorphism $\alpha$ of $N$ induces an automorphism $\hat{\alpha}$ of $\operatorname{Con}(N)$. Hence, there is an obvious group homomorphism $h:$ Aut $N \rightarrow \operatorname{Aut} \operatorname{Con}(N)$ mapping $\alpha$ to $\hat{\alpha}$. Classify those antilattices $N$ for which $h$ is an isomorphism.

Problem 8.3. Let us call a lattice $L$ transitive if for each pair of maximal chains $a$ and $b$ of $L$ there exists an automorphism of $L$ that maps one to the other one, i.e. it maps $a$ into $b$. We call an antilattice $N$ transitive, if its congruence lattice $\operatorname{Con}(N)$ is transitive. Classify transitive antilattices.

\section{Background}

For an antilattice $N$, a congruence $\theta$ is an equivalence relation on $N$ that is compatible with both $\wedge$ and $\vee$. Congruence relations form a lattice, $\operatorname{Con}(N)$.

\section{Joao Pita Costa}

Problem 9.1. Can we build on the coset structure of a skew distributive lattice to characterize skew Heyting algebras through their coset structure. And what can be said about the coset structure of skew Boolean algebras? Will it give us some insights on the respective dual spaces?

Problem 9.2. Can we always describe coset law-like identities that can determine a variety of skew lattices? Or are there varieties of skew lattices that do not permit such characterization?

Problem 9.3. The index theorems presented for some varieties of skew lattices in [3, 17] and [19] show a combinatorial perspective on these algebras as a direct consequence of their coset structure. Can we always obtain such results? What can they bring to the study of skew lattices?

For a concise background on the coset structure of several varieties of skew lattices and how they relate to each other, and to their index theorems, please refer to [18].

\section{Andreja Tepavčević}

Problem 10.1. Representation of algebraic lattices by a weak congruence lattice. All weak congruences form an algebraic lattice under inclusion. The diagonal relation (equality) has a particular role within weak congruence lattice. A 30-years unsolved problem [22] is as follows: if we take an algebraic lattice $L$ and pick an element a, under which conditions there is an algebra such that its weak congruence lattice is isomorphic to $L$ and the diagonal element corresponds to the fixed element a under this isomorphism. This is not possible for every element in a lattice, since this element should be codistributive and should satisfy some particular conditions. For further background please read [4] and [23].

Problem 10.2. Congruence lattice characterization. Characterize congruence lattices (and later weak congruence lattices) of skew lattices. It is well-known [8] that the congruence lattice of a lattice is distributive since there is the corresponding Mal'cev term. Is there a similar result for skew lattices? 


\section{Background}

Congruences are compatible equivalence relations. Weak congruences are compatible relations satisfying the same as congruences except they are not reflexive. Weak congruences are congruences on subalgebras of an algebra $A$ (equivalently - symmetric, transitive and compatible relations of $A$ ).

\section{Russ Woodroofe}

Problem 11.1. What is the correct universal algebra generalization of order congruence lattices? More broadly: find examples of comodernistic lattices!

\section{Background}

An element $m$ of a lattice $L$ is left-modular if for every pair $x \leq y$ of elements in $L$, we can write $x \vee m \wedge y$ without parentheses. That is, $m$ is left-modular if

$$
\forall x \leq y, \quad(x \vee m) \wedge y=x \wedge(m \wedge y)
$$

Left-modularity is a lattice-theoretic generalization of the Dedekind modular identity of group theory.

A lattice $L$ is comodernistic if every nontrivial interval $[a, b]$ of the lattice has a coatom that is left-modular in the interval. That is, for every $a<b$ in $L$, there is an $m$ covered by $b$, so that $m$ satisfies the left-modular relation with respect to every pair $a \leq x \leq y \leq b$. Jay Schweig and myself introduced comodernistic lattices in [20].

Comodernistic lattices generalize dual semimodular lattices, which can be defined as those lattices where every coatom of every interval is left-modular. Comodernistic lattices also generalize supersolvable lattices, which are those graded lattices which have a maximal chain consisting of left-modular elements. There are additional examples:

Example 11.2. The subgroup lattice of any finite solvable group is comodernistic. This follows from the fact that normal subgroups are left-modular, by the Dedekind modular identity.

Example 11.3. Similarly, the subalgebra lattice of any finite Lie algebra is comodernistic.

Example 11.4. The order congruence lattice $\mathcal{O}(P)$ of a finite poset $P$ consists of all the level sets of all the order preserving maps with domain $P$, ordered by refinement. Order congruence lattices are comodernistic, as can be seen in two steps:

(1) Let $\mu$ be the partition with a maximal element in a singleton block, and all other elements together in a big block. Then $\mu$ is left-modular.

(2) Intervals in $\mathcal{O}(P)$ are direct sums of order congruence lattices of quotients of subposets of $P$. Existence of modularity in coatoms of quotients follows from (1). Existence of left-modular coatoms is preserved by direct sum.

\section{Back to the Problem}

I'm interested in finding comodernistic lattices (or dually, modernistic lattices) in other settings. Perhaps there is a wider class of congruence lattices from universal algebra or similar which yields comodernistic lattices? 


\section{References}

[1] A. Bauer, K. Cvetko-Vah, M. Gehrke, S. J. van Gool and G. Kudryavtseva, A non-commutative Priestley duality, Topology Appl. 160 (2013), 1423-1438, doi:10.1016/j.topol.2013.05.012.

[2] I. Chajda, Congruence distributivity in varieties with constants, Arch. Math. (Brno) 22 (1986), 121-124, http://hdl. handle.net/10338.dmlcz/107253.

[3] K. Cvetko-Vah and J. P. Costa, On the coset laws for skew lattices, Semigroup Forum 83 (2011), 395-411, doi:10.1007/s00233-011-9325-7.

[4] G. Czédli, M. Erné, B. Šešelja and A. Tepavčević, Characteristic triangles of closure operators with applications in general algebra, Algebra Universalis 62 (2009), 399-418, doi:10.1007/ s00012-010-0059-2.

[5] D. G. Fitzgerald, Groupoids on a skew lattice of objects, Art Discrete Appl. Math. 2 (2019), \#P2.03, doi:10.26493/2590-9770.1342.109.

[6] M. Gehrke and S. J. van Gool, Sheaves and duality, J. Pure Appl. Algebra 222 (2018), 2164 2180, doi:10.1016/j.jpaa.2017.09.004.

[7] M. D. Gerhardts, Schrägverbände und Quasiordnungen, Math. Ann. 181 (1969), 65-73, doi: 10.1007/bf01351180.

[8] G. Grätzer, General Lattice Theory, Birkhäuser Verlag, Basel, 2nd edition, 2003.

[9] J. L. Gross and T. W. Tucker, Topological Graph Theory, Wiley-Interscience Series in Discrete Mathematics and Optimization, John Wiley \& Sons, New York, 1987.

[10] M. G. Jackson, General Algebra and its Applications 2013: problem session, Algebra Universalis 74 (2015), 9-16, doi:10.1007/s00012-015-0343-2.

[11] B. Jónsson, Algebras whose congruence lattices are distributive, Math. Scand. 21 (1967), 110121, doi:10.7146/math.scand.a-10850.

[12] G. Laslo and J. Leech, Green's equivalences on noncommutative lattices, Acta Sci. Math. (Szeged) 68 (2002), 501-533, http://pub.acta.hu/acta/ showCustomerArticle.action?id=2881\&dataObject Type=article.

[13] J. Leech, Skew Boolean algebras, Algebra Universalis 27 (1990), 497-506, doi:10.1007/ bf01188995.

[14] A. Malnič, R. Nedela and M. Škoviera, Lifting graph automorphisms by voltage assignments, European J. Combin. 21 (2000), 927-947, doi:10.1006/eujc.2000.0390.

[15] A. Malnič, T. Pisanski and A. Žitnik, The clone cover, Ars Math. Contemp. 8 (2015), 95-113, doi:10.26493/1855-3974.513.cbb.

[16] T. Pisanski, A classification of cubic bicirculants, Discrete Math. 307 (2007), 567-578, doi: 10.1016/j.disc.2005.09.053.

[17] J. Pita Costa, Coset laws for categorical skew lattices, Algebra Universalis 68 (2012), 75-89, doi:10.1007/s00012-012-0194-z.

[18] J. Pita Costa, On the Coset Structure of Skew Lattices, Ph.D. thesis, University of Ljubljana, Slovenia, 2012.

[19] J. Pita Costa and J. Leech, On the coset structure of distributive skew lattices, Art Discrete Appl. Math. 2 (2019), \#P2.05, doi:10.26493/2590-9770.1305.f45.

[20] J. Schweig and R. Woodroofe, A broad class of shellable lattices, Adv. Math. 313 (2017), 537563, doi:10.1016/j.aim.2017.04.007.

[21] W. Taylor, Characterizing Mal'cev conditions, Algebra Universalis 3 (1973), 351-397, doi: 10.1007/bf02945141. 
[22] G. Vojvodić and B. Šešelja, On the lattice of weak congruence relations, Algebra Universalis 25 (1988), 121-130, doi:10.1007/bf01229965.

[23] B. Šešelja, V. Stepanović and A. Tepavčević, A note on representation of lattices by weak congruences, Algebra Universalis 68 (2012), 287-291, doi:10.1007/s00012-012-0206-z. 